\title{
Antimicrobial Resistance, FlaA Sequencing, and Phylogenetic Analysis of Campylobacter Isolates from Broiler Chicken Flocks in Greece
}

\author{
George Natsos ${ }^{1}$, Niki K. Mouttotou ${ }^{2}$, Emmanouil Magiorkinis ${ }^{3}{ }^{\circledR}$, Anastasios Ioannidis ${ }^{4}\left({ }^{\circ}\right.$, Maria Magana ${ }^{4}$, \\ Stylianos Chatzipanagiotou ${ }^{5}$ and Konstantinos C. Koutoulis $1, * \mathbb{C}$
}

1 Department of Poultry Diseases, Veterinary Faculty, University of Thessaly, 43100 Karditsa, Greece; natsos@vet.uth.gr

2 Ministry of Rural Development and Foods, National Reference Laboratory for Salmonella and Antimicrobial Resistance, 34100 Chalkida, Greece; nmouttotou@minagric.gr

3 Department of Laboratory Haematology, General Hospital for Chest Diseases "Sotiria", 11527 Athens, Greece; mayiork@med.uoa.gr

4 Department of Nursing, Faculty of Human Movement and Quality of Life Sciences, University of Peloponnese, 23100 Sparta, Greece; tasobi@uop.gr (A.I.); mariamgn91@gmail.com (M.M.)

5 Department of Medical Biopathology, Medical School-Eginition Hospital, National and Kapodistrian University of Athens, 15772 Athens, Greece; schatzi@med.uoa.gr

* Correspondence: kkoutoulis@vet.uth.gr; Tel.: +30-693-743-7789

check for updates

Citation: Natsos, G.; Mouttotou, N.K.; Magiorkinis, E.; Ioannidis, A.; Magana, M.; Chatzipanagiotou, S.; Koutoulis, K.C. Antimicrobial Resistance, FlaA Sequencing, and Phylogenetic Analysis of Campylobacter Isolates from Broiler Chicken Flocks in Greece. Vet. Sci. 2021, 8, 68. https://doi.org/10.3390/ vetsci8050068

Academic Editor: Zuowei Wu

Received: 17 March 2021

Accepted: 19 April 2021

Published: 21 April 2021

Publisher's Note: MDPI stays neutral with regard to jurisdictional claims in published maps and institutional affiliations.

\begin{abstract}
Human campylobacteriosis caused by thermophilic Campylobacter species is the most commonly reported foodborne zoonosis. Consumption of contaminated poultry meat is regarded as the main source of human infection. This study was undertaken to determine the antimicrobial susceptibility and the molecular epidemiology of 205 Campylobacter isolates derived from Greek flocks slaughtered in three different slaughterhouses over a 14-month period. A total of $98.5 \%$ of the isolates were resistant to at least one antimicrobial agent. In terms of multidrug resistance, $11.7 \%$ of isolates were resistant to three or more groups of antimicrobials. Extremely high resistance to fluoroquinolones (89\%), very high resistance to tetracycline $(69 \%)$, and low resistance to macrolides (7\%) were detected. FlaA sequencing was performed for the subtyping of 64 C. jejuni and 58 C. coli isolates. No prevalence of a specific flaA type was observed, indicating the genetic diversity of the isolates, while some flaA types were found to share similar antimicrobial resistance patterns. Phylogenetic trees were constructed using the neighbor-joining method. Seven clusters of the C. jejuni phylogenetic tree and three clusters of the C. coli tree were considered significant with bootstrap values $>75 \%$. Some isolates clustered together were originated from the same or adjacent farms, indicating transmission via personnel or shared equipment. These results are important and help further the understanding of the molecular epidemiology and antimicrobial resistance of Campylobacter spp. derived from poultry in Greece.
\end{abstract}

Keywords: Campylobacter spp.; poultry; antimicrobial resistance; flaA typing; phylogenetic trees; Greece

\section{Introduction}

Campylobacter spp. are ubiquitous bacteria, able to colonize mucosal surfaces, usually the intestinal tract, of most mammalian and avian species [1,2]. Thermophilic Campylobacter spp. are essentially commensal in birds and insignificant for poultry health [3]. However, they are of high importance to food safety and public health, since they are recognized as the leading cause of bacterial foodborne diarrheal disease worldwide [1,4]. Birds carrying Campylobacter are asymptomatic colonizers without any clinical signs [5]. Broilers are considered Campylobacter free after hatching, since most evidence suggests that vertical transmission plays a minor role, if any [1], and, in general, broiler flocks remain 
Campylobacter free for the first two weeks (the so-called lag phase) [6]. This lag phase is likely to be an inherent property of the chick. An inhibitory effect produced by commensal organisms in the gut of young chicks [7], the presence of maternal antibodies, which may be protective and which decline by about 14 days of age [8], and antimicrobial treatment contribute to the existence of the lag phase. As chickens are coprophagic, fecal shedding is presumably an important factor in the dissemination of organisms around large broiler flocks once the first bird becomes colonized. Certainly, once flock colonization is detected, bird-to-bird transmission within flocks is extremely rapid, and the majority (up to 100\%) of birds in a positive flock are colonized within only a few days [6,9].

Consumption of poultry meat or ready-to-eat meat cross-contaminated by contact with raw poultry products constitutes the main risk factor for sporadic human infection $[1,10,11]$. Thus, control of campylobacteriosis is commonly focused on reducing the occurrence of Campylobacter in broiler meat [12]. C. jejuni is the predominant species isolated from poultry samples, followed by $C$. coli, with other Campylobacter species such as C. lari being less detected [13]. However, the predominance of $C$. coli has been reported in Greece $[14,15]$ and other southern European countries [13], which could be attributed to the differences in climatic conditions, environmental reservoirs, housing systems of broiler chickens, and age of slaughter between northern and southern Europe [16]. C. jejuni is, as well, considered responsible for the majority of human campylobacteriosis, followed by C. coli, and, rarely, by other emerging Campylobacter species, including C. concisus, C. ureolyticus, C. upsaliensis, and C. lari [4].

In the European Union (EU), campylobacteriosis has been the most commonly reported cause of human foodborne zoonoses since $2005[17,18]$. Antimicrobial treatment is usually not required, but effective treatment may shorten the duration of illness [19]. In cases where antimicrobial treatment is needed, macrolides (mostly erythromycin and azithromycin) and fluoroquinolones (e.g., ciprofloxacin) are considered as the first and second choices of antimicrobials, respectively $[20,21]$. Since a rapidly increasing proportion of Campylobacter strains worldwide have been found to be resistant to these antimicrobials, attention should be paid to choosing the most appropriate antimicrobial treatment [19]. Infection with antimicrobial-resistant Campylobacter may lead to suboptimal outcomes of antimicrobial treatments or even treatment failure [22]. Therefore, other antimicrobials such as gentamicin, carbapenems, and amoxicillin-clavulanic acid could be alternatively used for the treatment of systemic Campylobacter infections [23]. Transmission of antimicrobial resistance from food animals to humans can occur via the food chain. Therefore, food animals are a significant reservoir of antimicrobial-resistant zoonotic pathogens [24]. Consequently, the estimation of antimicrobial susceptibility of Campylobacter strains derived from animal samples is crucial. The World Health Organization, therefore, has published a list of critically important antimicrobials for human medicine, emphasizing the importance of prudent use of antimicrobials both in human and veterinary medicine [25].

Due to the impact of Campylobacter on public health, epidemiological investigations analyzing the clonality of the isolated strains are very important, in order to trace the sources and routes of transmission, to follow up the temporal and geographic distribution of important phenotypic characteristics, and to develop effective strategies for the control and prevention of the pathogen spread, especially inside the food chain $[26,27]$. The subtyping of clinical, animal, and food isolates remains an important requirement for epidemiological studies in order to (1) trace sources and routes of transmission of human infections; (2) identify and monitor, temporally and geographically, specific strains with important phenotypic characteristics; and (3) develop strategies to control organisms within the food chain [28]. Classical pulsed-field gel electrophoresis (PFGE) and amplified fragment length polymorphism (AFLP), as well as flaA typing based on the restriction analysis of PCR-amplified fragments or sequencing of the flagellin-encoding gene, have been described for Campylobacter [29-31]. Although, multilocus sequence typing (MLST) has been described as the gold standard method in this field, MLST is still time consuming and expensive and, therefore, not feasible for routine testing [30,31]. 
The aims of the present study were multiple: (1) to determine the antimicrobial resistance of Campylobacter isolates derived from Greek flocks in relation to common antimicrobial substances used in poultry practice and for human medicine, (2) to subtype them using the flaA gene sequencing typing technique, and (3) to perform a phylogenetic analysis in order to study their molecular epidemiology.

\section{Materials and Methods}

\subsection{Experimental Design}

The experimental procedure was conducted in commercial flocks. Therefore, an ethical approval from the University's Animal Ethics Committee was not required. Samples were collected from 142 slaughter batches, originating from 60 different poultry farms between February 2014 and March 2015 [15]. Caeca were randomly selected from 10 birds per batch during evisceration and pooled into a sterile bag. Neck skin samples of five birds from the processing line after chilling were also taken, using a clean pair of latex gloves and put into a sterile bag. After the sampling, the acquired samples were sent, in an insulated box containing ice packs to maintain a low temperature, within a few hours of the same day to Veterinary Laboratory of Chalkida, where bacteriological analyses were performed.

\subsection{Sample Analysis}

Campylobacter spp. recovered from the caecal contents using the technique of direct isolation, in which $10 \mu \mathrm{L}$ of each caecal sample, previously homogenized by adding Peptone Salt solution (Merck, Darmstadt, Germany), were plated on the selective medium, modified Charcoal Cefoperazone Deoxycholate Agar (mCCDA) (Oxoid, Dardilly, France), followed by incubation for $44 \pm 4 \mathrm{~h}$ at $41.5 \pm 1{ }^{\circ} \mathrm{C}$ under microaerobic conditions $\left(5 \% \mathrm{O}_{2}, 10 \% \mathrm{CO}_{2}\right.$, and $85 \% \mathrm{~N}_{2}$ ). For each positive plate, if necessary, up to five typical Campylobacter colonies were then subcultured onto plates of Columbia Blood Agar (Oxoid, Dardilly, France) for further characterization, in accordance with standard procedure of International Organization for Standardization (ISO) 10272-1 [32]. The flock was considered Campylobacter-positive, when at least one confirmed Campylobacter isolated from a colony yielded a positive result by PCR procedure.

For the recovery of Campylobacter from the skin of carcasses, the procedure described in ISO 10272 was followed. For the detection of Campylobacter, $10 \mathrm{~g}$ of neck skin was placed in a sterile bag and diluted 1:10 with selective pre-enrichment Bolton Broth solution (Oxoid, Dardilly, France). The mix was then homogenized for $1 \mathrm{~min}$ in a peristaltic homogenizer and the final suspension was incubated under microaerobic conditions for $4 \mathrm{~h}$ at $37^{\circ} \mathrm{C}$ and then for $44 \pm 4 \mathrm{~h}$ at $41.5 \pm 1^{\circ} \mathrm{C}$. Subsequently, $10 \mu \mathrm{L}$ of the suspension were plated onto mCCDA and Butzler (Oxoid, Dardilly, France) plates and followed by incubation for $44 \pm 4 \mathrm{~h}$ at $41.5 \pm 1{ }^{\circ} \mathrm{C}$. For each positive plate, up to five colonies typical of Campylobacter were subcultured onto Columbia Blood Agar plates for further characterization, according to standard method of ISO 10272-1:2006.

\subsection{Antimicrobial Susceptibility Testing}

For each Campylobacter-positive sample, antimicrobial susceptibility testing to ciprofloxacin, nalidixic acid, erythromycin, streptomycin, gentamicin, and tetracycline was performed. Antimicrobial disks for the disk diffusion method were obtained from Oxoid, Dardilly, France. Disk diffusion method in Mueller-Hinton agar enriched with 5\% defibrinated sheep's blood was performed. Sterile cotton-tipped swabs were used to inoculate broth culture diluted to match a $0.5 \mathrm{McFarland}$ turbidity standard onto Mueller-Hinton blood agar plates to produce a confluent lawn of bacterial growth. After the inoculum on the plates was dried, antimicrobial disks were distributed over the inoculated plates using an Antimicrobial Susceptibility testing Disk Dispencer (Oxoid, Dardilly, France). These plates were then incubated at $42{ }^{\circ} \mathrm{C}$ for $24 \mathrm{~h}$ under microaerobic conditions $\left(5 \% \mathrm{O}_{2}\right.$, $10 \% \mathrm{CO}_{2}$, and $85 \% \mathrm{~N}_{2}$ ). Isolates with insufficient growth after $24 \mathrm{~h}$ of incubation were re-incubated immediately and inhibition zone was read after a total of 40-48 h of incuba- 
tion. Campylobacter jejuni ATCC 33560 was used as a quality-control (QC) strain and the acceptable ranges of Clinical and Laboratory Standards Institute (CLSI M45) were followed. Since there were no antimicrobial susceptibility breakpoints for disk diffusion method specific with respect to Campylobacter for nalidixic acid, gentamicin, and streptomycin provided by CLSI M45, breakpoints of Enterobacteriaceae were used (CLSI M100). The concentrations of antimicrobial agents tested in this study along with the zone diameter breakpoints are shown in Table 1.

Table 1. Breakpoints of the disk diffusion method used to determine antimicrobial susceptibility of Campylobacter isolates.

\begin{tabular}{ccccc}
\hline \multirow{2}{*}{ Antimicrobial Agent } & \multicolumn{3}{c}{ Disk Concentration $(\boldsymbol{\mu g})$} & \multicolumn{2}{c}{ Zone Diameter Breakpoint $(\mathbf{m m})^{\mathbf{1}}$} \\
\cline { 3 - 5 } & & $\mathbf{S}$ & $\mathbf{I}$ & $\mathbf{R}$ \\
\hline Ciprofloxacin & 5 & $\geq 24$ & $21-23$ & $\leq 20$ \\
Erythromycin & 15 & $\geq 16$ & $13-15$ & $\leq 12$ \\
Tetracycline & 30 & $\geq 26$ & $23-25$ & $\leq 22$ \\
Nalidixic acid & 30 & $\geq 19$ & $14-18$ & $\leq 13$ \\
Gentamicin & 10 & $\geq 15$ & $13-14$ & $\leq 12$ \\
Streptomycin & 10 & $\geq 15$ & $12-14$ & $\leq 11$ \\
\hline
\end{tabular}

${ }^{1}$ Zone diameter breakpoints of ciprofloxacin, erythromycin, and tetracycline for Campylobacter spp. were recommended by the CLSI M45, whereas those of nalidixic acid, gentamicin, and streptomycin for Enterobacteriaceae were recommended by the CLSI M100. S, susceptible; I, intermediate; R, resistant.

\subsection{FlaA Sequencing}

A PCR procedure was performed on the DNA extracts of 122 Campylobacter isolates. The primers used (FLA4F and FLA630R) were composed by Eurofins Genomics, were in freeze-drying state, and were selected based on a study of Meinersmann et al. [33]. Sanger sequencing was performed in a 3130 Genetic Analyzer (Applied Biosystems Life Technologies Ltd., Paisley, UK). For the sequencing of the flaA gene, DNA STAR's Laser gene Evolution Suite software was used. All sequences were submitted to GenBank and issued accession numbers (MW713238-MW713296 for C. coli sequences and MW713297MW713360 for C. jejuni sequences).

\subsection{Phylogenetic Trees}

All available flaA sequences for C. jejuni and C. coli were downloaded from different geographic regions. For $C$. jejuni, the analysis involved 64 sequences isolated in our study plus 960 flaA reference sequences (RS) downloaded from the GenBank database. For C. coli, the numbers were 58 sequences plus 74 flaA reference sequences, respectively. Phylogenetic analysis was performed, estimating the genetic distances between sequences using TamuraNei model [34]. Phylogenetic trees were constructed using the neighbor-joining method and the reliability of phylogenetic clusters was assessed using bootstrapping analysis of 1000 copies. The trees were drawn to scale, with branch lengths in the same units as those of the evolutionary distances used to infer the phylogenetic trees. The alignment of all sequences was performed by Cluster W algorithm using the MEGA 5 version 5.0 software, while all positions containing gaps and missing data were manually edited.

\section{Results}

\subsection{Antimicrobial Resistance}

According to CLSI antimicrobial susceptibility breakpoints, $86.7 \%$ of Campylobacter isolates from caecal samples were classified as resistant to ciprofloxacin, $87.6 \%$ as resistant to nalidixic acid, and $77.1 \%$ as resistant to tetracycline. On the other hand, very low resistance to erythromycin $(7.6 \%)$ and streptomycin $(11.4 \%)$ and no resistance to gentamicin were found. Similar results came from antimicrobial resistance testing of neck skin samples (Table 2). The results of antimicrobial susceptibility in relation to the species of Campylobacter isolates are shown in Table 3. 
Table 2. Antimicrobial susceptibility patterns of Campylobacter spp., identified by the disk diffusion method, according to the sample tested ${ }^{1}$.

\begin{tabular}{|c|c|c|c|c|c|c|c|c|}
\hline \multirow{3}{*}{$\begin{array}{c}\text { Antimicrobial } \\
\text { Agent }\end{array}$} & \multicolumn{4}{|c|}{ Caecal Samples } & \multicolumn{4}{|c|}{ Neck Skin Samples } \\
\hline & \multicolumn{3}{|c|}{$\begin{array}{c}\text { No. of Campylobacter } \\
\text { Isolates }^{2}\end{array}$} & \multirow{2}{*}{$\begin{array}{c}\% \text { of } \\
\text { Resistant } \\
\text { Isolates }\end{array}$} & \multicolumn{3}{|c|}{$\begin{array}{c}\text { No. of Campylobacter } \\
\text { Isolates }^{2}\end{array}$} & \multirow{2}{*}{$\begin{array}{c}\% \text { of } \\
\text { Resistant } \\
\text { Isolates }\end{array}$} \\
\hline & $S$ & I & $\mathbf{R}$ & & $S$ & I & $\mathbf{R}$ & \\
\hline Ciprofloxacin & 14 & & 91 & 86.7 & 8 & & 92 & 92 \\
\hline Erythromycin & 97 & & 8 & 7.6 & 91 & 3 & 6 & 6 \\
\hline Tetracycline & 22 & 2 & 81 & 77.1 & 39 & & 61 & 61 \\
\hline Nalidixic acid & 13 & & 92 & 87.6 & 9 & 2 & 89 & 89 \\
\hline Gentamicin & 105 & & & 0 & 100 & & & 0 \\
\hline Streptomycin & 92 & 1 & 12 & 11.4 & 93 & & 7 & 7 \\
\hline
\end{tabular}

${ }^{1}$ The total number of Campylobacter isolates from caecal samples tested for antimicrobial resistance was 105 and from neck skin samples was 100. ${ }^{2}$ Number of susceptible (S), intermediate (I), and resistant (R) Campylobacter isolates identified by the disk diffusion method.

Table 3. Antimicrobial susceptibility patterns of Campylobacter isolates, identified by the disk diffusion method, according to the species ${ }^{1}$.

\begin{tabular}{|c|c|c|c|c|c|c|c|c|}
\hline \multirow{3}{*}{$\begin{array}{c}\text { Antimicrobial } \\
\text { Agent }\end{array}$} & \multicolumn{4}{|c|}{ Campylobacter Jejuni } & \multicolumn{4}{|c|}{ Campylobacter Coli } \\
\hline & \multicolumn{3}{|c|}{$\begin{array}{c}\text { No. of Campylobacter } \\
\text { Isolates }^{2}\end{array}$} & \multirow{2}{*}{$\begin{array}{c}\% \text { of } \\
\text { Resistant } \\
\text { Isolates }\end{array}$} & \multicolumn{3}{|c|}{$\begin{array}{c}\text { No. of Campylobacter } \\
\text { Isolates }^{2}\end{array}$} & \multirow{2}{*}{$\begin{array}{c}\% \text { of } \\
\text { Resistant } \\
\text { Isolates }\end{array}$} \\
\hline & $\mathbf{S}$ & $\mathbf{I}$ & $\mathbf{R}$ & & $S$ & I & $\mathbf{R}$ & \\
\hline Ciprofloxacin & 7 & & 95 & 93.1 & 15 & & 88 & 85.4 \\
\hline Erythromycin & 94 & & 8 & 7.8 & 94 & 3 & 6 & 5.8 \\
\hline Tetracycline & 29 & 1 & 72 & 70.6 & 32 & 1 & 70 & 68 \\
\hline Nalidixic acid & 7 & 1 & 94 & 92.2 & 15 & 1 & 87 & 84.5 \\
\hline Gentamicin & 102 & & & 0 & 103 & & & 0 \\
\hline Streptomycin & 90 & 1 & 11 & 10.8 & 94 & 1 & 8 & 7.7 \\
\hline
\end{tabular}

${ }^{1}$ The total number of Campylobacter jejuni was 102 and Campylobacter coli was 103. ${ }^{2}$ Number of susceptible (S), intermediate (I), and resistant (R) Campylobacter isolates identified by the disk diffusion method.

Only three strains were susceptible to all antimicrobial agents. Additionally, 13 out of 205 (6.3\%) Campylobacter isolates showed co-resistance to ciprofloxacin and erythromycin, whereas 24 out of $205(11.7 \%)$ were resistant to three or more groups of antimicrobials (i.e., fluoroquinolones, macrolides, tetracyclines, aminoglycosides).

\subsection{FlaA Sequencing}

A high degree of genetic diversity was revealed, with a total of 38 different nucleotide types that corresponded to 15 different peptide types. Peptide type 1 was the most predominant since it was recovered from 58 Campylobacter isolates. Of the isolates, 92.6\% (113 out of 122) showed exact match with the already registered ones in the international database, whereas $7.4 \%$ (9 out of 122) displayed partial match; namely, the isolates had a rate of homology though preserving different regions inside the sequences. Some isolates shared the same nucleotide and peptide type in an exact match with the registered types in the international database, suggesting the occurrence of clonality. Moreover, some of these isolates shared common antimicrobial profile (e.g., peptide type 1-DNA type 66).

\subsection{Phylogenetic Trees}

The phylogenetic trees of C. jejuni and C. coli isolates are shown in Figures 1a and 2a. Whereas most of the sequences found to be scattered inside the trees, seven clusters of the C. jejuni phylogenetic tree (Figure $1 \mathrm{~b}$ ) and three clusters of the $C$. coli tree (Figure $2 \mathrm{~b}$ ) were considered significant with bootstrap values $>75 \%$. 
Among the 13 C. jejuni isolates of the first cluster, eight shared the same DNA and peptide fla type, while two isolates (03FLA-33FLA) originated from the same poultry farm had the same antimicrobial profile. All eight isolates from the second cluster shared the same DNA and peptide fla type, while there were two pairs (21FLA-63FLA and 40FLA57FLA) that originated from the same farms and had similar antimicrobial profile. Likewise, in the third cluster, there were two isolates (20FLA-43FLA) that originated from the same farm and shared both identical DNA and peptide fla type and antimicrobial profile. All five isolates from the fourth cluster had the same DNA and peptide fla type and quite similar antimicrobial resistance. In the fifth cluster, there were three isolates (15FLA-27FLA-28FLA) that originated from two adjacent houses of the same farm and shared the same DNA and peptide fla type and antimicrobial profile. All seven clusters included reference sequences isolated from different regions (mainly USA, Europe, Tanzania, and Australia). However, no clear connection between them and the isolates of the current study could be made.

In the first cluster of $C$. coli phylogenetic tree, three of eight isolates (C60-C62-C63) originated from neighboring farms located in the same region and exhibited similar antimicrobial resistance patterns. The second cluster included only four isolates (C82-C83-C111C112), all of which originated from the same poultry farm and shared similar antimicrobial profiles. Almost all reference sequences in the first and third clusters originated from the USA, with the exception of one sequence that originated from Japan.

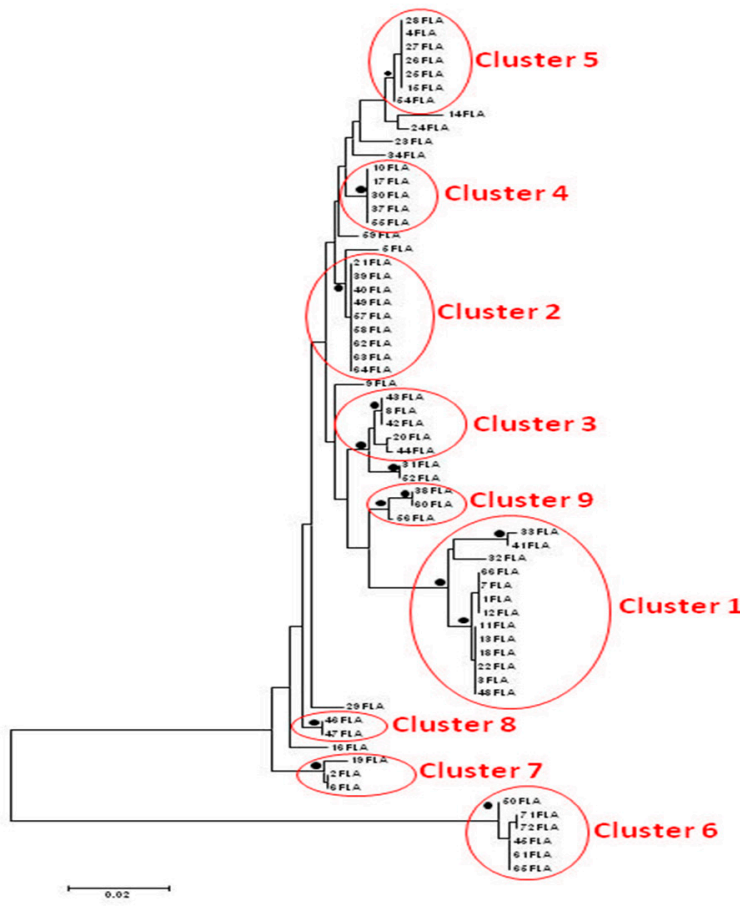

(a)

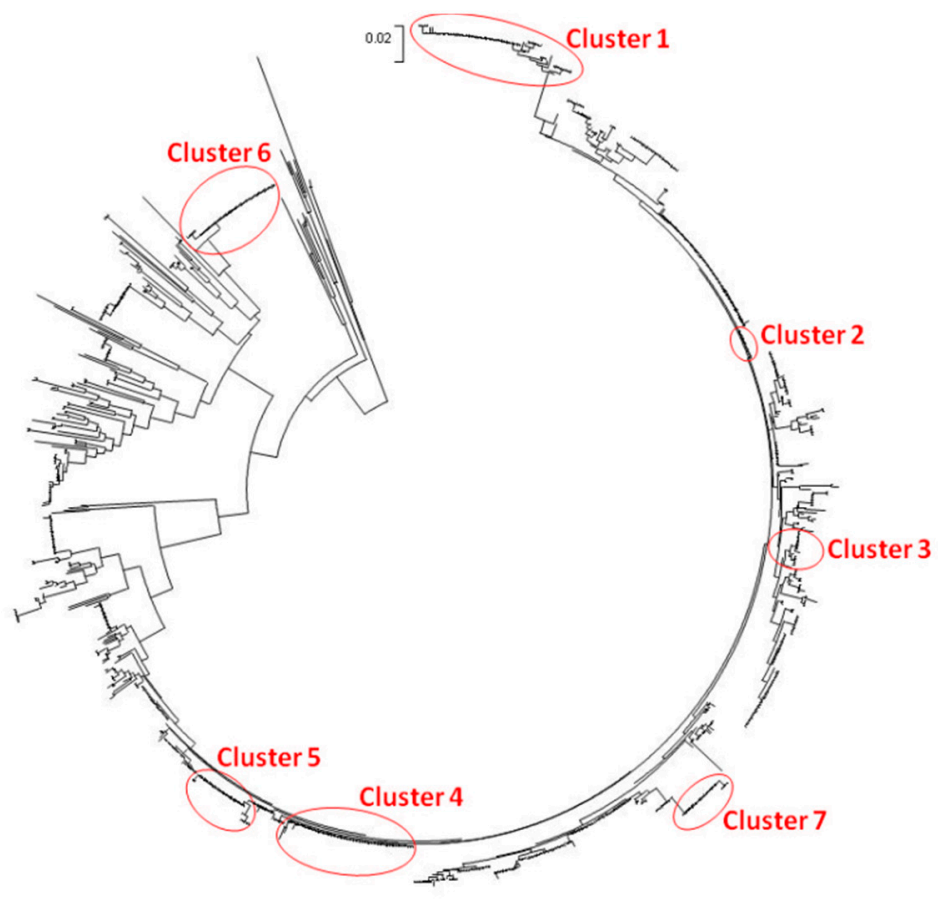

(b)

Figure 1. Campylobacter jejuni phylogenetic trees. Bullets represent clades, which had bootstrap values $>75 \%$ of permuted trees. (a) The analysis involved 64 sequences. Most of the sequences were organized in nine significant clusters supported with high bootstrap values. (b) The optimal tree with the sum of branch length $=4.34019783$ is shown. 


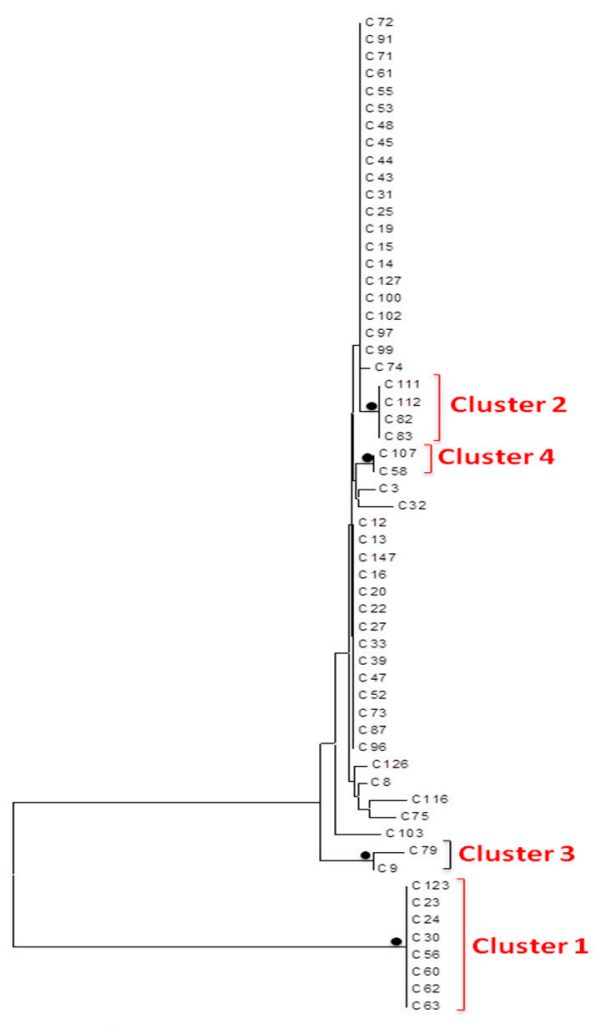

(a)

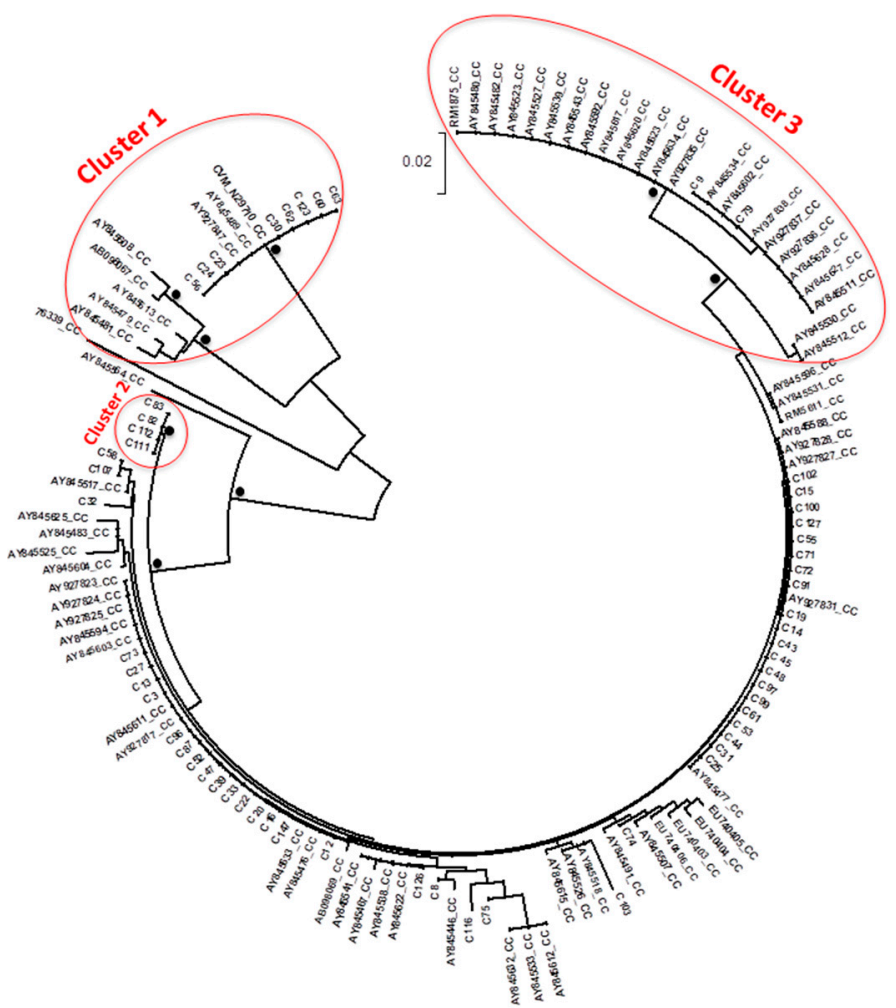

(b)

Figure 2. Campylobacter coli phylogenetic trees. Bullets represent clades, which had bootstrap values $>75 \%$ of permuted trees. (a) The optimal tree with the sum of branch length $=0.21165541$ is shown. The analysis involved 58 sequences. Most of the sequences were dispersed within the tree, whereas four significant sequence clusters were noticed. (b) The optimal tree with the sum of branch length $=0.54$ is shown.

\section{Discussion}

The results of our study regarding the antimicrobial resistance are consistent with other studies [20,35]. More specifically, high resistance to ciprofloxacin $(89.3 \%)$ and nalidixic acid $(88.3 \%)$ was observed. Similar results were submitted on the view of the obligatory monitoring and report of antimicrobial resistance by Greece in 2014, while the overall resistance to quinolones at the EU level was slightly lower [36]. Resistance to fluoroquinolones in Campylobacter spp. was firstly reported in the late 1980s and, since then, there is a continuous increase of resistance to fluoroquinolones [37]. It has been observed that resistance appeared simultaneously with the introduction of these agents in animal production and veterinary medicine [11,22]. Since campylobacteriosis is considered to be a zoonosis, the presence of resistant strains in the food chain also has an influence on human infections [11]. Moreover, it has been noted that the proportion of ciprofloxacin-resistant members of the genus Campylobacter in poultry meat is often strikingly similar to the proportion observed in human clinical cases [36]. However, the transmission of fluoroquinolone-resistant bacteria from food-producing animals to humans is difficult to prove, and a recent global report on surveillance of antimicrobial resistance emphasized the need to collect more data of the effects of antimicrobial resistance in foodborne bacteria and human health $[38,39]$. Besides their excessive use in agriculture, the use of fluoroquinolones for infections other than gastroenteritis, as well as "self-medication", are often causes of the observed resistance in developing countries [40]. Therefore, traveling to developing countries has been implied to be a risk factor for gaining an infection caused by a resistant Campylobacter strain. In the developed world, one reason behind fluoroquinolone resistance might also be their inappropriate empirical use in the treatment of human infections. Patients treated 
with fluoroquinolones were later found to carry bacteria resistant to these antimicrobial agents [41].

A low percentage $(6.8 \%)$ of Campylobacter among the strains recovered from caeca and neck skin samples was resistant to erythromycin. This result agrees with the respective ones of the EU survey [19]. However, the majority of these isolates revealed multi-antimicrobialresistance properties, a finding demonstrated in other studies, as well [42]. Resistance to erythromycin, as a rule, corresponds to cross resistance to other macrolides (for example, azithromycin and clarithromycin), as well as to related drugs of the group of lincosamides (in particular, to clindamycin) and streptogramins [43]. Resistance of Campylobacter spp. to macrolides has remained in low and stable levels for a long time. However, there is also evidence from some parts of the world that resistance rates to erythromycin and other macrolides in Campylobacter species are slowly increasing [44,45]. Since fluoroquinolone resistance is common, the macrolides have become important in the treatment of campylobacteriosis, resulting in the development of macrolide resistance [36]. Use of macrolides in animal production as therapeutic or growth-promoting agents has been considered to be a significant factor in the selection of erythromycin-resistant Campylobacter strains [46]. However, acquisition of erythromycin resistance in Campylobacter species is a stepwise process and requires prolonged exposure, in contrast to the rapidly evolving fluoroquinolone resistance [47]. Moreover, Hao et al. have shown that erythromycin-resistant Campylobacter strains display a fitness disadvantage when compared with susceptible Campylobacter strains, which may lead to a low frequency of macrolide resistance in clinical isolates [48].

Regarding the remaining antimicrobial agents, resistance of Campylobacter isolates to tetracycline was found to be remarkably high, especially in strains derived from caecal content. Similarly high resistance rates were observed in the recent report of EFSA and ECDC [19]. Tetracyclines can be used in the treatment of campylobacteriosis, except for children under 9 years of age [49]. However, tetracycline resistance has emerged also among Campylobacter species [36]. In Campylobacter spp. the most common tetracycline resistance mechanism is a plasmid-mediated ribosomal protecting protein, Tet $(\mathrm{O})$, encoded by the tet $(\mathrm{O})$ gene [50]. No resistance to gentamicin and low resistance to streptomycin were found. Quite similar results have been observed in most EU members states [19]. GuyardNicodème et al. [51] tested the susceptibility of $C$. jejuni strains derived from broiler meat products collected in retail outlets and found similar results with our study for tetracycline and gentamicin. The main mechanism of aminoglycoside resistance in Campylobacter spp. is via aminoglycoside-modifying enzymes, which are usually plasmid-borne [20]. Only three Campylobacter isolates showed complete susceptibility to all antimicrobial agents tested. Similar results were submitted by Greece in the frame of the EU survey [36]. On the other hand, $7.6 \%$ of $C$. jejuni and $5 \%$ of $C$. coli were co-resistant to ciprofloxacin and erythromycin. This fact is worrying since these antimicrobial classes constitute the cornerstone in treatment of severe human campylobacteriosis. Moreover, $13.3 \%$ of C. jejuni and $10 \%$ of C. coli strains showed multidrug resistance (MDR), defined as resistance or no-susceptibility to at least three antimicrobial classes-fluoroquinolones, macrolides, tetracyclines, or aminoglycosides [52]. The increase of multidrug-resistant Campylobacter strains has increased [53,54], posing a serious risk of treatment failures, since there are very few treatment alternatives of campylobacteriosis caused by multidrug-resistant strains [21]. This increase may reflect the overuse of different antimicrobial agents in veterinary medicine and, especially, in poultry production [11,39], as well as in human medicine, especially when administered without medical prescription [40].

In order to determine the antimicrobial resistance of Campylobacter, the disk diffusion method was used. Although the agar dilution method used to determine the minimal inhibitory concentration (MIC) is considered the standard antimicrobial susceptibility testing method for thermophilic Campylobacter species [31], it is a labor-intensive, time-consuming, and costly test [55]. On the other hand, the disk diffusion method is simple, inexpensive, and can provide reproducible results if it is conducted carefully with appropriate standardization and quality controls $[56,57]$. The latter method has been standardized 
by the CLSI. However, according to those standards, it should be used only as a screening method for resistance to erythromycin and ciprofloxacin; a disk diffusion zone of 6 $\mathrm{mm}$ (growth up to the edge of a 6-mm disk) indicates resistance, while any inhibition zone would require an MIC determination of susceptibility (CLSI M45). Due to the lack of breakpoints for the rest of the antibiotics, it was decided to use breakpoints of Enterobacteriacea provided by CLSI M100 [58]. This study revealed a high-level correlation between the standardized agar dilution method and the agar disk diffusion method for aminoglycosides, quinolone/fluoroquinolones, erythromycin, and tetracycline in evaluating the resistance of Campylobacter spp. Several comparisons of agreement between the disk diffusion method and other susceptibility testing methods for Campylobacter have been conducted over the years [57-61], some of which have concluded that disk diffusion method could be used as a reliable alternative method for the testing of susceptibility of Campylobacter spp. to ciprofloxacin and erythromycin $[57,58]$. On the other hand, the results of other studies are different and indicate the unreliability of this method and the need of further standardization $[60,61]$.

The selection of antimicrobials was done according to the published data concerning the widely used antimicrobial agents, both in poultry production and in the treatment of human campylobacteriosis, and followed the panel of antimicrobials from the EU protocol for harmonized monitoring of antimicrobial resistance in human Salmonella and Campylobacter isolates [62]. Campylobacter isolates from each positive sample were tested for resistance to ciprofloxacin, nalidixic acid, erythromycin, streptomycin, gentamicin, and tetracycline, as in the recent EU summary report [36].

Phylogenetic analysis of our strains using reference sequences highlighted seven clusters of $C$. jejuni isolates and three clusters of $C$. coli isolates in our study population. Almost all significant clusters included both sequences of the current cross-sectional study and reference sequences. No clear connection between our $C$. jejuni isolates and the reference sequences was found, even though most of the reference sequences originated from the USA. However, almost all reference sequences in the first and third clusters of C. coli originated from two surveys conducted in the USA. The first one dealt with isolates from retail chicken products and humans with gastroenteritis in central Michigan [63], while the second one dealt with isolates from the European CampyNet collection and National Antimicrobial Resistance Monitoring System, derived mostly from humans, chicken, cattle, and swine [64]. No safe conclusion could be drawn, though.

Some of the strains grouped in the same cluster and shared similar antimicrobial profile and fla types were derived from the same farms in different sampling time or from adjacent houses of the same farm. This finding indicates persistence of the infective strains in the house during turnaround time and further contamination of subsequent batches and/or infection of equipment and working clothes, leading to the spread of these strains from one house to another. Indeed, Campylobacter can be carried via boots and clothes of farm personnel and shared equipment between broiler houses of the same farm [65-67]. Moreover, the presence of colonized flocks has been found to be linked to the turnaround time in a broiler house. Periods of over 14 days can decrease the possibility of residual bacterial contamination [65], while the rapid flock turnover contributes to Campylobacter carryover with increased risk being reported if houses are restocked within nine days of depopulation [68]. In any case, the biosecurity and hygiene level should be maintained optimally during the empty time, as it is well known that an external reservoir can host multiple Campylobacter strains during the empty period, which will allow colonization of the new flock [66].

The presence of isolates with the same fla types and shared antimicrobial resistance patterns collected from different farms within a close distance in the same region could be attributed to vehicles that visit different farms in the same day without applying adequate disinfection, such as feed delivery trucks, vehicles for collection of litter and dead birds, or transport from the hatchery and to processing plants, which act as mechanical vectors and allow the transmission of these strains from each farm to another. Farm personnel 
and equipment (e.g., feed trucks) can carry Campylobacter between broiler houses and onto subsequent or neighboring farms [65]. Although feed is not seen as a high-risk Campylobacter contaminant within the broiler house, since the low water activity of the dry feed does not permit Campylobacter survival [67], it can be a vehicle for horizontal transmission into the broiler house [11]. Hald et al. [69] showed that the incidence of Campylobacter was lower in farms that feed homegrown wheat compared to farms that are dependent on external supplies. Jonsson et al. [70] found that livestock and broiler farms with flocks positive for Campylobacter spp. within a few kilometers' distance constitute significant risks for colonization in broiler flocks. Furthermore, live bird crates being contaminated with Campylobacter from previous (or other) flocks are reintroduced on the farm during catching, and quite often these crates undergo inadequate washing at the slaughterhouse [65]. Crates can carry identical genotypes of microorganisms that originated from broiler flock and abattoirs, which suggests that transport crates are responsible for contamination during transport to slaughter or they could contribute to the Campylobacter colonization of broiler houses [71].

\section{Conclusions}

In conclusion, the cross-sectional study carried out in Greece produced valuable results concerning the antimicrobial resistance and the molecular epidemiology of Campylobacter spp. in poultry production countrywide. High resistance to fluoroquinolones and tetracycline and low resistance to macrolides and aminoglycosides was found. A high genetic diversity was found, while some specific flaA types were found to share similar antimicrobial-resistance patterns. Phylogenetic analysis of the isolates revealed eight clusters of $C$. jejuni and three clusters of $C$. coli. Some isolates clustered together originated from the same or adjacent farms, indicating transmission via personnel or shared equipment. No clear connection between the reference sequences used and the isolates of the current study was found. These results are of high importance and constitute the foundation in understanding the molecular epidemiology and susceptibility patterns of Campylobacter spp. derived from poultry in Greece.

Author Contributions: All authors helped to draft the manuscript and participated in its design. Conceptualization, K.C.K., N.K.M. and G.N.; investigation, G.N.; formal analysis, G.N., E.M., A.I. and M.M.; resources, K.C.K.; writing-original draft preparation, G.N.; writing-review and editing, N.K.M., S.C., A.I. and E.M.; visualization, G.N. and E.M.; supervision, K.C.K., N.K.M. and A.I.; project administration, K.C.K.; funding acquisition, K.C.K. All authors have read and agreed to the published version of the manuscript.

Funding: The project "Campylobacter spp. in the broiler food chain: Measuring and monitoring the risk for public health" is granted by the "General Secretariat of Research and Technology"-Ministry of Education and Religious Affairs-under the "Bilateral Cooperation R\&T Program between Greece and France".

Institutional Review Board Statement: Ethical review and approval were waived for this study, due to the execution of the experimental protocol in commercial poultry flocks.

Informed Consent Statement: Not applicable.

Data Availability Statement: The data presented in this study are available on reasonable request from the corresponding author.

Acknowledgments: The authors would like to thank the Ministry of Rural Development and Foods, National Reference Laboratory of Salmonella and Antimicrobial Resistance of Chalkida, for its contribution in isolation, detection, enumeration, and PCR speciation of Campylobacter spp. in caeca and poultry carcass.

Conflicts of Interest: The authors declare no conflict of interest. The funders had no role in the design of the study; in the collection, analyses, or interpretation of data; in the writing of the manuscript, or in the decision to publish the results. 


\section{References}

1. Natsos, G.; Koutoulis, K.C.; Sossidou, E.; Chemaly, M.; Mouttotou, N.K. Campylobacter spp. infection in humans and poultry. J. Hell. Vet. Med. Soc. 2016, 67, 65-77. [CrossRef]

2. Rossler, E.; Olivero, C.; Soto, L.P.; Frizzo, L.S.; Zimmermann, J.; Rosmini, M.R.; Sequeira, G.J.; Signorini, M.L.; Zbrun, M.V. Prevalence, genotypic diversity and detection of virulence genes in thermotolerant Campylobacter at different stages of the poultry meat supply chain. Int. J. Food Microbiol. 2020, 326, 108641. [CrossRef]

3. Sahin, O.; Kassem, I.I.; Shen, Z.; Lin, J.; Rajashekara, G.; Zhang, Q. Campylobacter in poultry: Ecology and potential interventions. Avian Dis. 2015, 59, 185-200. [CrossRef]

4. Kaakoush, N.O.; Castano-Rodriguez, N.; Mitchell, H.M.; Man, S.M. Global epidemiology of Campylobacter infection. Clin. Microbiol. Rev. 2015, 28, 687-720. [CrossRef] [PubMed]

5. Lee, M.D.; Newell, D.G. Campylobacter in poultry: Filling an ecological niche. Avian Dis. 2006, 50, 1-9. [CrossRef] [PubMed]

6. Newell, D.G.; Fearnley, C. Sources of Campylobacter colonization in broiler chickens. Appl. Environ. Microbiol. 2003, 69, 4343-4351. [CrossRef] [PubMed]

7. Schoeni, J.L.; Doyle, M.P. Reduction of Campylobacter jejuni colonization of chicks by cecum-colonizing bacteria producing anti-C. jejuni metabolites. Appl. Environ. Microbiol. 1992, 58, 664-670. [CrossRef] [PubMed]

8. Sahin, O.; Zhang, Q.; Meitzler, J.C.; Harr, B.S.; Morishita, T.Y.; Mohan, R. Prevalence, antigenic specificity, and bactericidal activity of poultry anti-Campylobacter maternal antibodies. Appl. Environ. Microbiol. 2001, 67, 3951-3957. [CrossRef] [PubMed]

9. Shreeve, J.E.; Toszeghy, M.; Pattison, M.; Newell, D.G. Sequential spread of Campylobacter infection in a multipen broiler house. Avian Dis. 2000, 44, 983-988. [CrossRef]

10. EFSA. EFSA Panel on Biological Hazards (BIOHAZ). Scientific Opinion on Quantification of the risk posed by broiler meat to human campylobacteriosis in the EU. EFSA J. 2010, 8, 89. [CrossRef]

11. Silva, J.; Leite, D.; Fernandes, M.; Mena, C.; Gibbs, P.A.; Teixeira, P. Campylobacter spp. as a Foodborne Pathogen: A Review. Front. Microbiol. 2011, 2, 200. [CrossRef] [PubMed]

12. Hue, O.; Le Bouquin, S.; Laisney, M.J.; Allain, V.; Lalande, F.; Petetin, I.; Rouxel, S.; Quesne, S.; Gloaguen, P.Y.; Picherot, M.; et al. Prevalence of and risk factors for Campylobacter spp. contamination of broiler chicken carcasses at the slaughterhouse. Food Microbiol. 2010, 27, 992-999. [CrossRef]

13. EFSA. Analysis of the baseline survey on the prevalence of Campylobacter in broiler batches and of Campylobacter and Salmonella on broiler carcasses in the EU, 2008, Part A: Campylobacter and Salmonella prevalence estimates. EFSA J. 2010, 8, 100. [CrossRef]

14. Marinou, I.; Bersimis, S.; Ioannidis, A.; Nicolaou, C.; Mitroussia-Ziouva, A.; Legakis, N.J.; Chatzipanagiotou, S. Identification and antimicrobial resistance of Campylobacter species isolated from animal sources. Front. Microbiol. 2012, 3, 58. [CrossRef]

15. Natsos, G.; Mouttotou, N.K.; Magiorkinis, E.; Ioannidis, A.; Rodi-Burriel, A.; Chatzipanagiotou, S.; Koutoulis, K.C. Prevalence of and risk factors for Campylobacter spp. colonization of broiler chicken flocks in Greece. Foodborne Pathog. Dis. 2020, 17, 679-686. [CrossRef]

16. EFSA. Analysis of the baseline survey on the prevalence of Campylobacter in broiler batches and of Campylobacter and Salmonella on broiler carcasses, in the EU, 2008; Part B: Analysis of factors associated with Campylobacter colonisation of broiler batches and with Campylobacter contamination of broiler carcasses; and investigation of the culture method diagnostic characteristics used to analyse broiler carcass samples. EFSA J. 2010, 8, 132. [CrossRef]

17. EFSA. The Community Summary Report on trends and sources of zoonoses, zoonotic agents, antimicrobial resistance and foodborne outbreaks in the European Union in 2005. EFSA J. 2006, 94, 288.

18. EFSA; ECDC. The European Union One Health 2018 Zoonoses Report. EFSA J. 2019, 17, 276.

19. EFSA; ECDC. The European Union Summary Report on Antimicrobial Resistance in zoonotic and indicator bacteria from humans, animals and food in 2017/2018. EFSA J. 2020, 18, 166.

20. Iovine, N.M. Resistance mechanisms in Campylobacter jejuni. Virulence 2013, 4, 230-240. [CrossRef]

21. Yang, Y.; Feye, K.M.; Shi, Z.; Pavlidis, H.O.; Kogut, M.; Ashworth, A.J.; Ricke, S.C. A historical review on antibiotic resistance of foodborne Campylobacter. Front. Microbiol. 2019, 10, 1509. [CrossRef]

22. Engberg, J.; Neimann, J.; Nielsen, E.M.; Aerestrup, F.M.; Fussing, V. Quinolone-resistant Campylobacter infections: Risk factors and clinical consequences. Emerg. Infect. Dis. 2004, 10, 1056-1063. [CrossRef] [PubMed]

23. Dai, L.; Sahin, O.; Grover, M.; Zhang, Q. New and alternative strategies for the prevention, control, and treatment of antibioticresistant Campylobacter. Transl. Res. 2020, 223, 76-88. [CrossRef] [PubMed]

24. Moore, J.E.; Barton, M.D.; Blair, I.S.; Corcoran, D.; Dooley, J.S.; Fanning, S.; Kempf, I.; Lastovica, A.J.; Lowery, C.J.; Matsuda, M.; et al. The epidemiology of antibiotic resistance in Campylobacter. Microbes Infect. 2006, 8, 1955-1966. [CrossRef] [PubMed]

25. WHO. Critically Important Antimicrobials for Human Medicine, 6th Revision; WHO: Geneva, Switzerland, 2019.

26. Sheppard, S.K.; Colles, F.; Richardson, J.; Cody, A.J.; Elson, R.; Lawson, A.; Brick, G.; Meldrum, R.; Little, C.L.; Owen, R.J.; et al. Host association of Campylobacter genotypes transcends geographic variation. Appl. Environ. Microbiol. 2010, 76, 5269-5277. [CrossRef]

27. Ioannidou, V.; Ioannidis, A.; Magiorkinis, E.; Bagos, P.; Nicolaou, C.; Legakis, N.; Chatzipanagiotou, S. Multilocus sequence typing (and phylogenetic analysis) of Campylobacter jejuni and Campylobacter coli strains isolated from clinical cases in Greece. BMC Res. Notes 2013, 6, 359. [CrossRef] [PubMed] 
28. Ioannidis, A.; Nicolaou, C.; Legakis, N.J.; Ioannidou, V.; Papavasileiou, E.; Voyatzi, A.; Chatzipanagiotou, S. Genotyping of human Campylobacter jejuni isolates in Greece by pulsed-field gel electrophoresis. Mol. Diagn. Ther. 2006, 10, 391-396. [CrossRef]

29. Wassenaar, T.M.; Newell, D.G. Genotyping of Campylobacter spp. Appl. Environ. Microbiol. 2000, 66, 1-9. [CrossRef]

30. Natsos, G.; Mouttotou, N.K.; Ahmad, S.; Kamran, Z.; Ioannidis, A.; Koutoulis, K.C. The genus Campylobacter: Detection and isolation methods, species identification \& typing techniques. J. Hell. Vet. Med. Soc. 2019, 70, 12.

31. Korczak, B.M.; Zurfluh, M.; Emler, S.; Kuhn-Oertli, J.; Kuhnert, P. Multiplex strategy for multilocus sequence typing, fla typing, and genetic determination of antimicrobial resistance of Campylobacter jejuni and Campylobacter coli isolates collected in Switzerland. J. Clin. Microbiol. 2009, 47, 1996-2007. [CrossRef]

32. ISO. Microbiology of Food and Animal Feeding Stuffs-Horizontal Method for Detection and Enumeration of Campylobacter spp. Part 1: Detection Method; [ISO 10272-1:2006]; International Organization for Standardization: Geneva, Switzerland, 2006.

33. Meinersmann, R.J.; Helsel, L.O.; Fields, P.I.; Hiett, K.L. Discrimination of Campylobacter jejuni isolates by fla gene sequencing. J. Clin. Microbiol. 1997, 35, 2810-2814. [CrossRef]

34. Tamura, K.; Nei, M. Estimation of the number of nucleotide substitutions in the control region of mitochondrial DNA in humans and chimpanzees. Mol. Biol. Evol. 1993, 10, 512-526. [CrossRef] [PubMed]

35. Fraqueza, M.J.; Martins, A.; Borges, A.C.; Fernandes, M.H.; Fernandes, M.J.; Vaz, Y.; Bessa, R.J.; Barreto, A.S. Antimicrobial resistance among Campylobacter spp. strains isolated from different poultry production systems at slaughterhouse level. Poult. Sci. 2014, 93, 1578-1586. [CrossRef] [PubMed]

36. EFSA; ECDC. The European Union summary report on antimicrobial resistance in zoonotic and indicator bacteria from humans, animals and food in 2014. EFSA J. 2016, 14, 207. [CrossRef]

37. Sproston, E.L.; Wimalarathna, H.M.L.; Sheppard, S.K. Trends in fluoroquinolone resistance in Campylobacter. Microb. Genom. 2018, 4. [CrossRef] [PubMed]

38. WHO. Antimicrobial Resistance: Global Report on Surveillance; WHO: Geneva, Switzerland, 2014.

39. Thanner, S.; Drissner, D.; Walsh, F. Antimicrobial resistance in agriculture. mBio 2016, 7, e02227-15. [CrossRef]

40. Coker, A.O.; Isokpehi, R.D.; Thomas, B.N.; Amisu, K.O.; Obi, C.L. Human campylobacteriosis in developing countries. Emerg. Infect. Dis. 2002, 8, 237-244. [CrossRef]

41. Bacon, D.J.; Alm, R.A.; Burr, D.H.; Hu, L.; Kopecko, D.J.; Ewing, C.P.; Trust, T.J.; Guerry, P. Involvement of a plasmid in virulence of Campylobacter jejuni 81-176. Infect. Immun. 2000, 68, 4384-4390. [CrossRef]

42. Wieczorek, K.; Kania, I.; Osek, J. Prevalence and antimicrobial resistance of Campylobacter spp. isolated from poultry carcasses in Poland. J. Food Prot. 2013, 76, 1451-1455. [CrossRef] [PubMed]

43. Efimochkina, N.R.; Stetsenko, V.V.; Sheveleva, S.A. Formation of the resistance of Campylobacter jejuni to macrolide antibiotics. Bull. Exp. Biol. Med. 2020, 169, 351-356. [CrossRef]

44. Bae, W.; Kaya, K.N.; Hancock, D.D.; Call, D.R.; Park, Y.H.; Besser, T.E. Prevalence and antimicrobial resistance of thermophilic Campylobacter spp. from cattle farms in Washington State. Appl. Environ. Microbiol. 2005, 71, 169-174. [CrossRef] [PubMed]

45. Vlieghe, E.R.; Jacobs, J.A.; Van Esbroeck, M.; Koole, O.; Van Gompel, A. Trends of norfloxacin and erythromycin resistance of Campylobacter jejuni/Campylobacter coli isolates recovered from international travelers, 1994 to 2006. J. Travel Med. 2008, 15, 419-425. [CrossRef] [PubMed]

46. Ladely, S.R.; Harrison, M.A.; Fedorka-Cray, P.J.; Berrang, M.E.; Englen, M.D.; Meinersmann, R.J. Development of macrolideresistant Campylobacter in broilers administered subtherapeutic or therapeutic concentrations of tylosin. J. Food Prot. 2007, 70, 1945-1951. [CrossRef]

47. Lin, J.; Yan, M.; Sahin, O.; Pereira, S.; Chang, Y.J.; Zhang, Q. Effect of macrolide usage on emergence of erythromycin-resistant Campylobacter isolates in chickens. Antimicrob. Agents Chemother. 2007, 51, 1678-1686. [CrossRef] [PubMed]

48. Hao, H.; Dai, M.; Wang, Y.; Peng, D.; Liu, Z.; Yuan, Z. 23S rRNA mutation A2074C conferring high-level macrolide resistance and fitness cost in Campylobacter jejuni. Microb. Drug Resist. 2009, 15, 239-244. [CrossRef] [PubMed]

49. Moore, J.E.; Corcoran, D.; Dooley, J.S.; Fanning, S.; Lucey, B.; Matsuda, M.; McDowell, D.A.; Megraud, F.; Millar, B.C.; O’Mahony, R.; et al. Campylobacter. Vet. Res. 2005, 36, 351-382. [CrossRef]

50. Gibreel, A.; Tracz, D.M.; Nonaka, L.; Ngo, T.M.; Connell, S.R.; Taylor, D.E. Incidence of antibiotic resistance in Campylobacter jejuni isolated in Alberta, Canada, from 1999 to 2002, with special reference to tet(O)-mediated tetracycline resistance. Antimicrob. Agents Chemother. 2004, 48, 3442-3450. [CrossRef]

51. Guyard-Nicodème, M.; Rivoal, K.; Houard, E.; Rose, V.; Quesne, S.; Mourand, G.; Rouxel, S.; Kempf, I.; Guillier, L.; Gauchard, F.; et al. Prevalence and characterization of Campylobacter jejuni from chicken meat sold in French retail outlets. Int. J. Food Microbiol. 2015, 203, 8-14. [CrossRef] [PubMed]

52. Magiorakos, A.P.; Srinivasan, A.; Carey, R.B.; Carmeli, Y.; Falagas, M.E.; Giske, C.G.; Harbarth, S.; Hindler, J.F.; Kahlmeter, G.; Olsson-Liljequist, B.; et al. Multidrug-resistant, extensively drug-resistant and pandrug-resistant bacteria: An international expert proposal for interim standard definitions for acquired resistance. Clin. Microbiol. Infect. 2012, 18, 268-281. [CrossRef] [PubMed]

53. Chen, X.; Naren, G.W.; Wu, C.M.; Wang, Y.; Dai, L.; Xia, L.N.; Luo, P.J.; Zhang, Q.; Shen, J.Z. Prevalence and antimicrobial resistance of Campylobacter isolates in broilers from China. Vet. Microbiol. 2010, 144, 133-139. [CrossRef]

54. Qin, S.S.; Wu, C.M.; Wang, Y.; Jeon, B.; Shen, Z.Q.; Wang, Y.; Zhang, Q.; Shen, J.Z. Antimicrobial resistance in Campylobacter coli isolated from pigs in two provinces of China. Int. J. Food Microbiol. 2011, 146, 94-98. [CrossRef] 
55. McDermott, P.F.; Bodeis-Jones, S.M.; Fritsche, T.R.; Jones, R.N.; Walker, R.D. Broth microdilution susceptibility testing of Campylobacter jejuni and the determination of quality control ranges for fourteen antimicrobial agents. J. Clin. Microbiol. 2005, 43, 6136-6138. [CrossRef] [PubMed]

56. Potz, N.A.; Mushtaq, S.; Johnson, A.P.; Henwood, C.J.; Walker, R.A.; Varey, E.; Warner, M.; James, D.; Livermore, D.M. Reliability of routine disc susceptibility testing by the British Society for Antimicrobial Chemotherapy (BSAC) method. J. Antimicrob. Chemother. 2004, 53, 729-738. [CrossRef] [PubMed]

57. Gaudreau, C.; Girouard, Y.; Gilbert, H.; Gagnon, J.; Bekal, S. Comparison of disk diffusion and agar dilution methods for erythromycin, ciprofloxacin, and tetracycline susceptibility testing of Campylobacter coli and for tetracycline susceptibility testing of Campylobacter jejuni subsp. jejuni. Antimicrob. Agents Chemother. 2008, 52, 4475-4477. [CrossRef] [PubMed]

58. Luangtongkum, T.; Morishita, T.Y.; El-Tayeb, A.B.; Ison, A.J.; Zhang, Q. Comparison of antimicrobial susceptibility testing of Campylobacter spp. by the agar dilution and the agar disk diffusion methods. J. Clin. Microbiol. 2007, 45, 590-594. [CrossRef]

59. McGill, K.; Kelly, L.; Madden, R.H.; Moran, L.; Carroll, C.; O’Leary, A.; Moore, J.E.; McNamara, E.; O’Mahony, M.; Fanning, S.; et al. Comparison of disc diffusion and epsilometer (E-test) testing techniques to determine antimicrobial susceptibility of Campylobacter isolates of food and human clinical origin. J. Microbiol. Methods 2009, 79, 238-241. [CrossRef] [PubMed]

60. van der Beek, M.T.; Claas, E.C.; Mevius, D.J.; van Pelt, W.; Wagenaar, J.A.; Kuijper, E.J. Inaccuracy of routine susceptibility tests for detection of erythromycin resistance of Campylobacter jejuni and Campylobacter coli. Clin. Microbiol. Infect. 2010, 16, 51-56. [CrossRef]

61. Lehtopolku, M.; Kotilainen, P.; Puukka, P.; Nakari, U.M.; Siitonen, A.; Eerola, E.; Huovinen, P.; Hakanen, A.J. Inaccuracy of the disk diffusion method compared with the agar dilution method for susceptibility testing of Campylobacter spp. J. Clin. Microbiol. 2012, 50, 52-56. [CrossRef] [PubMed]

62. ECDC. EU Protocol for Harmonised Monitoring of Antimicrobial Resistance in Human Salmonella and Campylobacter Isolates; ECDC: Stockholm, Sweden, 2016.

63. Fitch, B.R.; Sachen, K.L.; Wilder, S.R.; Burg, M.A.; Lacher, D.W.; Khalife, W.T.; Whittam, T.S.; Young, V.B. Genetic diversity of Campylobacter sp. isolates from retail chicken products and humans with gastroenteritis in Central Michigan. J. Clin. Microbiol. 2005, 43, 4221-4224. [CrossRef]

64. Meinersmann, R.J.; Phillips, R.W.; Hiett, K.L.; Fedorka-Cray, P. Differentiation of Campylobacter populations as demonstrated by flagellin short variable region sequences. Appl. Environ. Microbiol. 2005, 71, 6368-6374. [CrossRef]

65. Newell, D.G.; Elvers, K.T.; Dopfer, D.; Hansson, I.; Jones, P.; James, S.; Gittins, J.; Stern, N.J.; Davies, R.; Connerton, I.; et al. Biosecurity-based interventions and strategies to reduce Campylobacter spp. on poultry farms. Appl. Environ. Microbiol. 2011, 77, 8605-8614. [CrossRef]

66. Ellis-Iversen, J.; Ridley, A.; Morris, V.; Sowa, A.; Harris, J.; Atterbury, R.; Sparks, N.; Allen, V. Persistent environmental reservoirs on farms as risk factors for Campylobacter in commercial poultry. Epidemiol. Infect. 2012, 140, 916-924. [CrossRef]

67. Sibanda, N.; McKenna, A.; Richmond, A.; Ricke, S.C.; Callaway, T.; Stratakos, A.C.; Gundogdu, O.; Corcionivoschi, N. A review of the effect of management practices on Campylobacter prevalence in poultry farms. Front. Microbiol. 2018, 9, 2002. [CrossRef] [PubMed]

68. Battersby, T.; Whyte, P.; Bolton, D.J. The pattern of Campylobacter contamination on broiler farms; external and internal sources. J. Appl. Microbiol. 2016, 120, 1108-1118. [CrossRef] [PubMed]

69. Hald, B.; Wedderkopp, A.; Madsen, M. Thermophilic Campylobacter spp. in Danish broiler production: A cross-sectional survey and a retrospective analysis of risk factors for occurrence in broiler flocks. Avian Pathol. 2000, 29, 123-131. [CrossRef]

70. Jonsson, M.E.; Chriel, M.; Norstrom, M.; Hofshagen, M. Effect of climate and farm environment on Campylobacter spp. colonisation in Norwegian broiler flocks. Prev. Vet. Med. 2012, 107, 95-104. [CrossRef] [PubMed]

71. Hastings, R.; Colles, F.M.; McCarthy, N.D.; Maiden, M.C.; Sheppard, S.K. Campylobacter genotypes from poultry transportation crates indicate a source of contamination and transmission. J. Appl. Microbiol. 2011, 110, 266-276. [CrossRef] [PubMed] 